\title{
Radiation-Free Drill Guidance in Interlocking of Intramedullary Nails
}

\author{
Benoit Diotte $^{1}$, Pascal Fallavollita ${ }^{1}$, Lejing Wang $^{1}$, Simon Weidert ${ }^{2}$, \\ Peter-Helmut Thaller ${ }^{2}$, Ekkehard Euler ${ }^{2}$, and Nassir Navab ${ }^{1}$ \\ 1 Chair for Computer Aided Medical Procedures (CAMP), TU Munich, Germany \\ 2 Trauma Surgery Department, Klinikum Innenstadt, LMU Munich, Germany
}

\begin{abstract}
Intramedullary nailing is a technically demanding procedure which involves an excessive amount of X-ray acquisitions; one study lists as many as 48 to successfully complete the procedure. In this work, a novel low cost radiation-free drilling guide is designed to assist surgeons in completing the distal locking procedure without any X-ray acquisitions. Using an augmented reality fluoroscope that coregisters optical and X-ray images, we exploit solely the optical images to detect the drilling guide in order to estimate the tip position in real-time in X-ray. We tested over 200 random drill guide poses showing a mean tip-estimation error of $1.72 \pm 0.7 \mathrm{~mm}$ which is significantly robust and accurate for the interlocking. In a preclinical study on dry bone phantom, three expert surgeons successfully completed the interlocking 56 out of 60 trials with no X-ray acquisition for guidance and an average time of $2 \mathrm{~min}$.
\end{abstract}

\section{Introduction}

Modern trauma and orthopedic surgeries such as fracture reduction and osteosynthesis use $\mathrm{C}$-arm X-ray or standard X-ray radiography for interventional guidance, especially in minimally invasive surgery. As X-ray is displayed in 2D, the surgeon relies on their skill set to mentally transfer the bone configuration shown in the image onto the patient location. This requires added mental effort and may lead to distraction, possibly resulting in procedural errors compromising quality of surgery. Tibial fractures are among the most common lower limb injuries to be treated by an orthopedic \& trauma surgeon. In the early 1990s, tibial fractures accounted for 77.000 hospitalizations per year. Today, the incidence has increased to approximately 500.000 cases in the United States per year. On average, almost 26 tibia fractures occur per 100.000 population per year 1 . Intramedullary nailing is a common surgical procedure mostly used in fracture reduction of the tibial and femoral shaft. After successful insertion of the nail into the medullary canal, the nail has to be fixed in its position in order to prevent rotation and dislocation by inserting screws perpendicular to the nail through the provided proximal and distal holes inside the nails shaft.

${ }^{1}$ Epidemiology at http://emedicine.medscape.com/article/

1248857-overview\#a0199 
The insertion of the screws near the entrance point of the nail is achieved using an aiming bow attached to the nail. In the distal part of the nail, locking is commonly performed freehand with a radiolucent drill attachment or similar aiming devices. Various techniques and devices developed for facilitating locking procedures are reviewed in [7]. The conclusions from this work are that interlocking of intramedullary nails is a technically demanding procedure. Several ingenious computer aided methods and devices were developed recently, e.g. robot based guide positioning [8] and 3D optical tracking [1] however these have not yet gained worldwide acceptance in clinical practice due to the higher learning curve and additional cumbersome hardware involved in the operating room. The amount of radiation exposure is excessive due to the intricacy of drilling a screw inside a $5 \mathrm{~mm}$ nailing hole solely by X-ray image acquisition and guidance.

Intraoperative Radiation Exposure: Much experimental work has been performed to evaluate the amount of radiation exposure to surgeons during interlocking procedures. Müller et al. [2] showed that the average X-ray time per procedure, mainly spent with distal locking, was $4.63 \mathrm{~min}$ and the primary surgeon received a $2.02 \mathrm{mSv}$ mean radiation exposure to his hand. On the other hand, Suhm et al. showed that when using a computer aided surgery navigation system, the radiation time exposure decreased from $108 \mathrm{sec}$ to $7 \mathrm{sec}$, but took significantly longer operation time from $13.7 \mathrm{~min}$ to $17.9 \mathrm{~min}$ when compared to a standard mobile C-arm fluoroscope [5]. In a more recent study by Rohilla et al., authors document that the average number of images taken for the complete procedure to be 48.27 [4. In an effort to reduce radiation exposure, Navab et al. have attempted an augmented reality (AR) fluoroscopy system for orthopedic surgery that allows to facilitate the surgical procedure and to reduce the radiation exposure. They augment a regular mobile $\mathrm{C}$-arm by a video camera and a X-ray/video image overlay [3]. Through a mirror construction and one time calibration of the device, the acquired X-ray images are coregistered with the video images without any further calibration or registration during the intervention. The surgeon can thus visualize on the same video both soft tissue and bone position. Such system allows them to better plan the scar positioning and the screw insertion point. Moreover, the surgeon can better target using the real-time video view where the X-ray image is taken, and therefore avoid unnecessary radiation. This technology has been described to reduce radiation; a decrease from $17.05 \pm 4.61 \mathrm{X}$-ray shots to $9.85 \pm 3.10$ using the system in [3. However, two immediate limitations of the overlay are observed. First, none of these works use the optical video image effectively as the optics provides a simple raw image to the surgeon; in other words, they often revert back to the $\mathrm{X}$-ray image for decision making and navigation completion. Secondly, the instrument tip (i.e. scalpel tip, drill tip, etc.) is not visible to the surgeon when it is positioned inside the patient anatomy of interest.

Contribution: In this paper, a radiation-free guide is designed and used to enable surgeons to complete the interlocking of intramedullary nailing procedure 


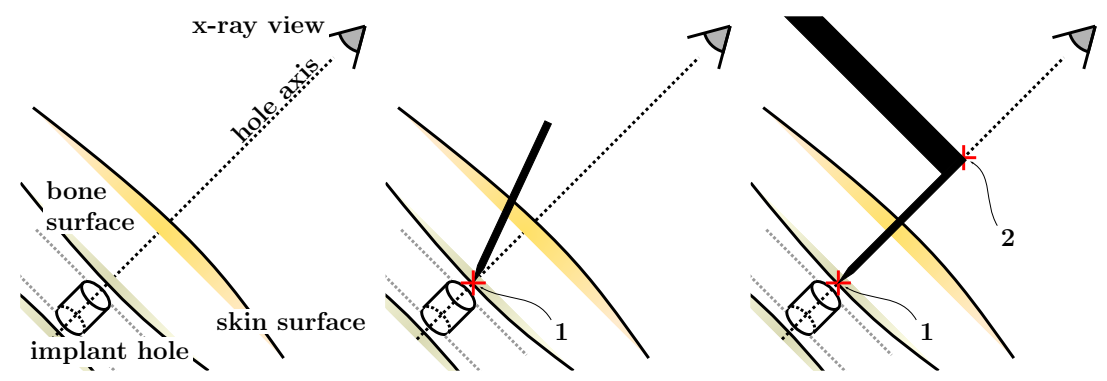

Fig. 1. (Left) The down-beam positioning technique for distal locking aligns the hole axis of the nail with the camera center to guide the drilling process. (Center) The center punch marks point 1. (Right) The drill top is aligned on the axis at point 2 . Points 1 and 2 are required to be kept on the axes by the surgeon in order to guarantee the success of drilling.

without any X-ray acquisitions. Motivated from the technologies in [3, for the very first time we propose to use the optical video images for full procedure guidance and navigation, thereby becoming the first such interlocking system using single view optical video input data. The radiation-free guide is detected by the optical camera and its tip is measured in real-time and displayed to the surgeon in both the X-ray and video images. In contrast to commercially available optical tracking tools we need no additional light sources or navigation base, and the cameras data is not solely dedicated to navigation. The camera is viewing inside the $\mathrm{C}$-arm working volume, usually free of occlusions. Our hypothesis is the complex interlocking of intramedullary nails procedure will be simplified. Via a computer-simulation and dry bone phantom study, we evaluate the precision and success rate of the interlocking of intramedullary nails.

\section{Methodology}

\subsection{The Surgical Workflow in Interlocking of Intramedullary Nails}

The typical distal locking workflow (WF) is divided into seven surgical steps [6]

WF 1) X-ray Positioning: Moving the C-arm on top of the patient and ends after the X-ray image shows the distal part of the nail inside the bone.

WF 2) Adjustment of Hole: Position the C-arm until the hole is perfectly round in order to allow for orthogonal drilling (i.e. down-beam positioning, fig. 1) WF 3) Skin Incision: Finding the incision position and cutting the skin. The correct incision position is confirmed by images showing the scalpel tip located inside the locking hole on the X-ray image or using augmented fluoroscopy as in 3 .

WF 4) Center Punch: Alignment of a Schanz screw with the target hole. Then, with the help of a hammer, a small dimple is formed on the bone surface in which 
the tip of the drill will be positioned. This step is required to prevent slipping of the drill. To ensure alignment X-ray images are acquired.

WF 5) Alignment of the Tip of the Drill: Alignment of the drill bit with the target hole. It ends when X-ray images show that the projection of the tip is located inside the circle of the target hole.

WF 6) Drilling: Drilling the bone until the drill bit passes through the locking hole of the nail and the bone cortex on the other side. It ends after confirmation by X-rays.

WF 7) Locking Screw Insertion: Inserting a locking screw into the hole. Xray is required to confirm the success of the insertion, which indicates the end of the procedure.

The challenge for the surgeon lies in aligning the drill along the down the beam axis to ensure a successful drilling process (fig. 1). This is achieved using an average of $9.15 \pm 6.65 \mathrm{X}$-ray images [6] in WF 4,WF 6] Our objective is to eliminate X-ray guidance completely during these steps by designing a radiationfree drilling guide.

\subsection{Radiation-Free Drill Guide Model}

We modified a $200 \mathrm{~mm}$ long, $5 \mathrm{~mm}$ diameter Schanz screw by fixing seven fluorescent coated spherical markers (fig. 2). The top ball has the largest size at $2.0 \mathrm{~cm}$. An upper branch containing 3 spherical markers is attached to the pin at position B. These three spherical markers have a size of $1.2 \mathrm{~cm}$ in diameter. A lower branch containing 3 spherical markers is attached to the pin at position C. These three spherical markers have a size of $1.0 \mathrm{~cm}$ in diameter. The reason for choosing different sized markers was for the correct ordering between the top ball, and upper, lower branch markers. The distances on the upper branch are: ab: $3.0 \mathrm{~cm}$, ac: $5.7 \mathrm{~cm}$, aB: $8.9 \mathrm{~cm}$. The lower branch has the same dimensions. Lastly, the metrics for the main axis of the Schanz screw are: $\mathbf{A B}: 4.7 \mathrm{~cm}, \mathbf{A C}$ : $10.7 \mathrm{~cm}$, AD: $18.8 \mathrm{~cm}$.
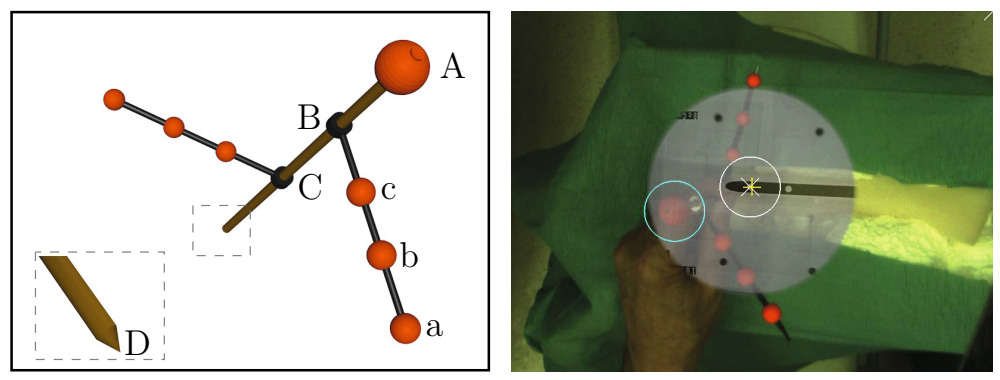

Fig. 2. (Left) The design of the radiation-free drill guide. (Right) The overlay of X-ray and video image. The target hole is bounded by a white circle marked with an $(\mathrm{x})$ for its center. The estimated tip position for the radiation-free drill guide is visualized by a yellow $(+)$ icon. 
Tip Estimation Using the Cross-Ratio. Having collinear markers detected in an image we are able to compute the tip-position of the radiation-free drill guide. Given the 3D geometry of our instrument, (i.e. the position of the distal end of the guide with respect to the other markers) we compute the tip based on the cross-ratio:

$$
\begin{gathered}
d_{A D}=\frac{\left(S \cdot d_{A B}\right)-d_{A C}}{S-1} \text { where } S=\frac{\text { crossratio } \cdot d_{A C}}{d_{A B}} \\
\text { crossratio }=\frac{A B \cdot C D}{A C \cdot B D}
\end{gathered}
$$

Here $d_{i j}$ are the image distances between the markers $i$ and $j$ respectively. The same idea is applied to estimate the image position of $\mathrm{B}$ and $\mathrm{C}$ on the main axis of the pin from the upper and lower branches; using the three collinear balls on each branch.

\section{Real-Time Detection of the Seven Spherical Markers in Video Image.}

We observed that using red fluorescent pigmentation peaked the saturation channel of HSV in such a way that thresholding the S image with a very high cutoff value results in the spherical markers being seen by the video camera as bright ellipses (i.e. close to a circular shape), which can be easily segmented. From the binary image all contours are extracted using the OpenCV library. In a postprocessing step we filter those contours having a low compactness value and those having a smaller area than a threshold (i.e. in our setup the default values are 0.5 for the compactness and $100 \mathrm{px}$ for the area). For all contours being retained by the filtering routine, the sub-pixel centroids are computed based on grayscale image moments. From the possible blob candidates we select the largest blob as being the most likely candidate for the top ball of the radiation free drill guide. From the remaining blobs, we choose two disjoint sets yielding an optimal least squares line fitting and least size variance. We now have the seven best estimates for the spherical marker center locations. From equation (1), we solve for B and $\mathrm{C}$ points located on the main axis of the Schanz screw by applying the same side axis cross ratio to the 2 branches. For each set we get 2 possible solutions (i.e. because cross ratio is based on a ball ordering process, and we don't have any point correspondences). To rectify this, we find the combination of estimated points that have the smallest distance to each other (i.e. assign them as possible $\mathrm{B}$ and $\mathrm{C}$ points). Lastly, we order the $\mathrm{B}$ and $\mathrm{C}$ combination on the main axis according to their distances from the top ball on a fitted line. Having ABC we can estimate the tip-position of the radiation-free drill guide (fig. (2). A note: we observe that turning the white balancing off in video stabilizes the blob detection based on our fluorescent colored markers.

\subsection{Quantification Experiments for Tip Estimation Accuracy}

We first performed an experiment to quantify the accuracy of the estimated tip position. We print a binary circle with $5 \mathrm{~mm}$ diameter on a sheet of paper 
that is placed about $20 \mathrm{~cm}$ away and parallel to the image intensifier. The image center of the circle is extracted with sub-pixel accuracy from the video image; defining ground-truth of the tip image position. We physically fix the tip of pin at the center of the circle for all pose variations. We rotated the radiationfree drill guide about the tip position to generate 200 video images of random different poses. We computed the difference between the estimated tip position and ground-truth to quantify the accuracy of the tip detection. The metric error in $\mathrm{mm}$ can be calculated from image error in px, since the ratio between px and $\mathrm{mm}$ is known. The rotational values spanned a cone angle range between $\pm 30^{\circ}$.

\subsection{Dry Bone Phantom Experiment}

In this pre-clinical phantom study, we used dry bones having similar shape and size, and common surgical instruments, including an AO radiolucent drill attachment, and a $10 \mathrm{~mm}$ solid Titanium Femoral Nail (Synthes, Oberdorf, Switzerland). The dry bones were placed inside a plastic box in a horizontal position and then fixed on the $\mathrm{C}$-arm intensifier. In order to simulate the rigidity of a normal leg, the dry bones were fixed inside the box. Each dry bone was drilled open from the proximal joint and the nail was inserted in the medullary cavity of the bone prior to the beginning of the experiment (fig. [3).

Visualization in X-ray and Video Image. For our method, we employ the system calibration method described in [3] in order to align the projection geometry of an X-ray and video image. The estimated tip of the radiation-free drill guide is directly registered to the X-ray images. The target hole of the nail seen in X-ray is bounded by a white circle and marked with an $\mathrm{x}$ for its center. The radiation-free drill guide tip is marked with a yellow + and the top ball of the guide is bounded with a blue circle (fig. 2). The visualization colors and details were suggested by the surgeons participating in our study. The AR fluoroscopy system used was built by attaching a video camera (Flea2, from Point Grey Research Inc., Vancouver, BC, Canada) and mirror construction, covered by an X-ray source housing, to a mobile C-arm (Powermobile, isocentric
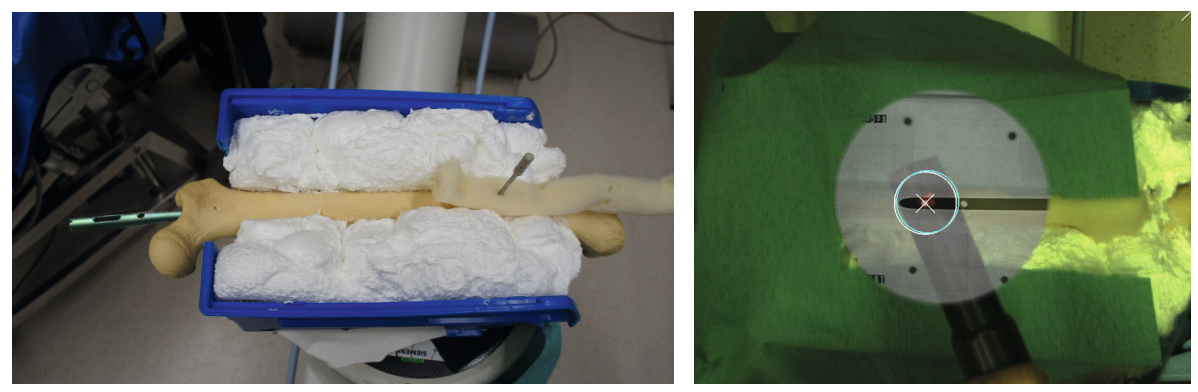

Fig. 3. (Left) The dry bone phantom setup with a nail inside the bone. (Right) The top ball of the drill guide is bounded by a blue circle. 
C-arm, from Siemens Healthcare, Erlangen, Germany). The mean error of the calibration computed on about 200 control points was $1.59 \pm 0.87 \mathrm{px}$ with a maximum error of 5.02 ; approximately $0.5 \mathrm{~mm}$ on the plane of the calibration pattern.

Surgical Protocol for Radiation-Free Interlocking. Two expert surgeons and one resident surgeon from the orthopedic and trauma surgery department participated in a total of 60 interlocking of intramedullary nails. 30 trials were performed with video and X-ray overlay visualization, and the remaining trials were performed by looking at augmented X-ray images. To begin with, an X-ray image of the nail within the dry bone was acquired along the down the beam position. The target entry point of the hole was automatically thresholded via a circular Hough transform. Then by visualizing the X-ray and video overlay, the surgeons maneuver the radiation-free drill guide until the tip was superimposed on the center of the target hole. The guide is then positioned along the down beam position prior to center punching. Using a hammer, the bone is notched by hammering down on top of our guide. Lastly, the drill tip is positioned on the notch by the surgeon and the drill top is detected in real-time in video and maneuvred until it is superimposed with the target center in X-ray (fig. 3). The surgeon lastly drills through the nail hole.

\section{Results and Discussion}

For the quantification study with 200 trials and $\pm 30^{\circ}$ cone angle rotation of the radiation free guide we obtain the following errors for tip estimation when compared to ground-truth: $3.93 \pm 1.6 \mathrm{px}$ which is equivalent to $1.72 \pm 0.7 \mathrm{~mm}$; $57 \%$ of the samples were below the mean error, $98 \%$ below $4 \mathrm{~mm}$. These errors indicate the accuracy and robustness of our detection and tip estimation algorithm and are within the clinical tolerances for interlocking procedures since the hole diameter of the nail is $5 \mathrm{~mm}$. For the preclinical dry bone phantom study, a success rate of $93 \%$ (56 out of 60 trials) was achieved by the surgeons. The average time for completion was $2 \mathrm{~min}$. No X-ray images were acquired or requested outside of one X-ray needed to confirm whether the drill missed or hit the hole of the nail. Therefore, by detecting the radiation-free drill guide only in video, the surgeons effectively guided the interlocking workflow steps WF 4.WF 6 defined in 2.1. This is considered the first attempts of using video guidance for augmented reality fluoroscopy systems as in [3]. Of the 4 failed trials, one was due to the nail moving out of position when the surgeon hammered the radiation-free drill guide to notch the bone. This was a design issue for our phantom setup. The three surgeons confirmed that in clinical practice the interlocking nail is fixed by external jigs and that the nail would not move out of position. The remaining 3 failed trials were attributed to the resident surgeon. Interestingly, when compared to the two expert surgeons, the resident surgeon did not perform a deep enough notch on the bone surface during the center punching step, thereby not being able to position properly the drill tip prior to drilling. He 
remedied his approach accordingly and succeeded in the remaining trials. The final results in both studies indicate that our radiation-free drill guide has the potential to eliminate a large number of X-ray acquisitions to complete the interlocking of intramedullary nails procedure. The immediate feedback received by the three surgeons was listing a potential orthopedic and trauma procedures that can immediately benefit from such a guide: interlocking nailing of humeral shaft fractures or plate osteosynthesis fixation.

\section{Conclusions}

In this paper, a radiation-free guide is designed and used to assist surgeons in completing the interlocking of intramedullary nailing procedure without additional X-ray acquisitions. This is the very first video guided study for completing the interlocking procedure. Our radiation-free guide has its tip estimated in real-time and displayed to the surgeon at all instances regardless if they are visualizing only augmented X-ray images. Our hypothesis is that the complex interlocking of intramedullary nails procedure will be simplified by our elegant radiation-free solution. Our results demonstrate potential for this.

\section{References}

1. Leloup, T., El Kazzi, W., Schuind, F., Warzee, N.: A novel technique for distal locking of intramedullary nail based on two non-constrained fluoroscopic images and navigation. IEEE Transactions on Medical Imaging 27(9), 1202-1212 (2008)

2. Müller, L.P., Suffner, J., Wenda, K., Mohr, W., Rommens, P.M.: Radiation exposure to the hands and the thyroid of the surgeon during intramedullary nailing. Injury 29(6), 461-468 (1998)

3. Navab, N., Heining, S.M., Traub, J.: Camera augmented mobile c-arm (camc): Calibration, accuracy study and clinical applications. IEEE Transactions on Medical Imaging 29(7), 1412-1423 (2009)

4. Rohilla, R., Singh, R., Magu, N., Devgan, A., Siwach, R., Sangwan, S.: Simultaneous use of cannulated reamer and schanz screw for closed intramedullary femoral nailing. ISRN Surg. (2011) (published online)

5. Suhm, N., Messmer, P., Zuna, I., Jacob, L.A., Regazzoni, P.: Fluoroscopic guidance versus surgical navigation for distal locking of intramedullary implants. a prospective, controlled clinical study. Injury 35(6), 567-574 (2004)

6. Wang, L., Landes, J., Weidert, S., Blum, T., von der Heide, A., Euler, E., Navab, N.: First Animal Cadaver Study for Interlocking of Intramedullary Nails under Camera Augmented Mobile C-arm. In: Navab, N., Jannin, P. (eds.) IPCAI 2010. LNCS, vol. 6135, pp. 56-66. Springer, Heidelberg (2010)

7. Whatling, G., Nokes, L.: Literature review of current techniques for the insertion of distal screws into intramedullary locking nails. Injury 37(2), 109-119 (2006)

8. Yaniv, Z., Joskowicz, L., Member, S.: Precise robot-assisted guide positioning for distal locking of intramedullary nails. IEEE Transactions on Medical Imaging 24(5), 624-635 (2005) 